\title{
EFFICIENT DESIGN INVESTIGATION OF A TURBOFAN IN DISTORTED INLET CONDITIONS
}

\author{
Dr. Benjamin Godard \\ Aerodynamics, Aeroelasticity \\ and Acoustics Department \\ Onera \\ Meudon, France \\ benjamin.godard@onera.fr
}

\author{
Edouard De Jaeghere \\ Safran Aircraft Engines \\ Moissy-Cramayel, France \\ edouard.de-jaeghere \\ @safrangroup.com
}

\author{
Pr. Nicolas Gourdain \\ Université de Toulouse \\ Institut Supérieur de l'Aéronautique \\ et de l'Espace (ISAE) \\ Toulouse, France \\ nicolas.gourdain@isae.fr
}

\section{ABSTRACT}

Designing a turbofan to operate in distorted inlet conditions is an issue of growing interest. In such conditions however, fan design can be computationally challenging. Indeed, subject to neither axi-symmetrical nor periodic inlet conditions, computations must be carried out throughout the whole circumferential domain i.e. $360^{\circ}$. Besides, the classical CFD approach implies the use of URANS computations so as to capture the distortion transfer across the fan stage. Eventually, computations are too onerous to be used in design loops. In this context, this paper presents a methodology to effectively assess a fan blade design domain in distorted conditions. This methodology is based on a body-force source term approach formulated in order to accurately recreate deviations, loads and losses across the fan stage. It notably enables to gain two orders in terms of restitution time and thus the possibility to use optimization tools. The design domain of this study is based on variations of profile chord, blade leading and trailing edges angles applied at two different relative heights. A Latin Hypercube Sampling (LHS) associated with a meta-model based on Radial Basis Functions (RBF) enables to assess the impact of geometric variations on performance and operability. Although this study emphasizes that some modeling improvements are still necessary, it also demonstrates the potential of the body-force methodology to conduct fan design when subject to inlet distortion.

\author{
NOMENCLATURE \\ BLI Boundary Layer Ingestion \\ LHS Latin Hypercube Sampling \\ RBF Radial Basis Functions \\ NSGA2 Non-dominated Sorting GA 2 \\ UHBR Ultra High Bypass Ratio \\ $a_{s} \quad$ Sound velocity $[\mathrm{m} / \mathrm{s}]$ \\ $b \quad$ Blockage factor $[-]$ \\ c $\quad$ Blade chord length $[m]$ \\ $c_{x} \quad$ Blade axial chord length $[m]$ \\ $\bar{c}_{x} \quad$ Normalized Blade axial chord length [\%] \\ $e_{x} \quad$ Blade axial thickness $[m]$ \\ $e_{t} \quad$ Total energy $[\mathrm{J} / \mathrm{kg}]$ \\ $H \quad$ Blade to blade staggered spacing $[\mathrm{m}]$ \\ $h \quad$ Relative height $[-]$ \\ $h_{t} \quad$ Stagnation specific enthalpy $[\mathrm{J} / \mathrm{kg}]$ \\ $\vec{f} \quad$ Body-forces $[\mathrm{N} / \mathrm{kg}]$ \\ $f_{n} \quad$ Body-force component normal to $\vec{w}[N / k g]$ \\ $f_{p} \quad$ Body-force component parallel to $\vec{w}[N / k g]$ \\ $\bar{f}_{\theta} \quad$ Circumferentially averaged and normalized tangential \\ force [\%] \\ $K_{p 0} \quad$ Friction drag coefficient at maximum efficiency [-] \\ $M \quad$ Absolute Mach number [-] \\ $M_{\text {rel }} \quad$ Relative Mach number [-] \\ $M_{x} \quad$ Axial Mach number [-] \\ $\dot{m} \quad$ Massflow rate $[\mathrm{kg} / \mathrm{s}]$ \\ $\dot{m}_{\text {corr }} \quad$ Corrected massflow rate $[\mathrm{kg} / \mathrm{s}]$
}




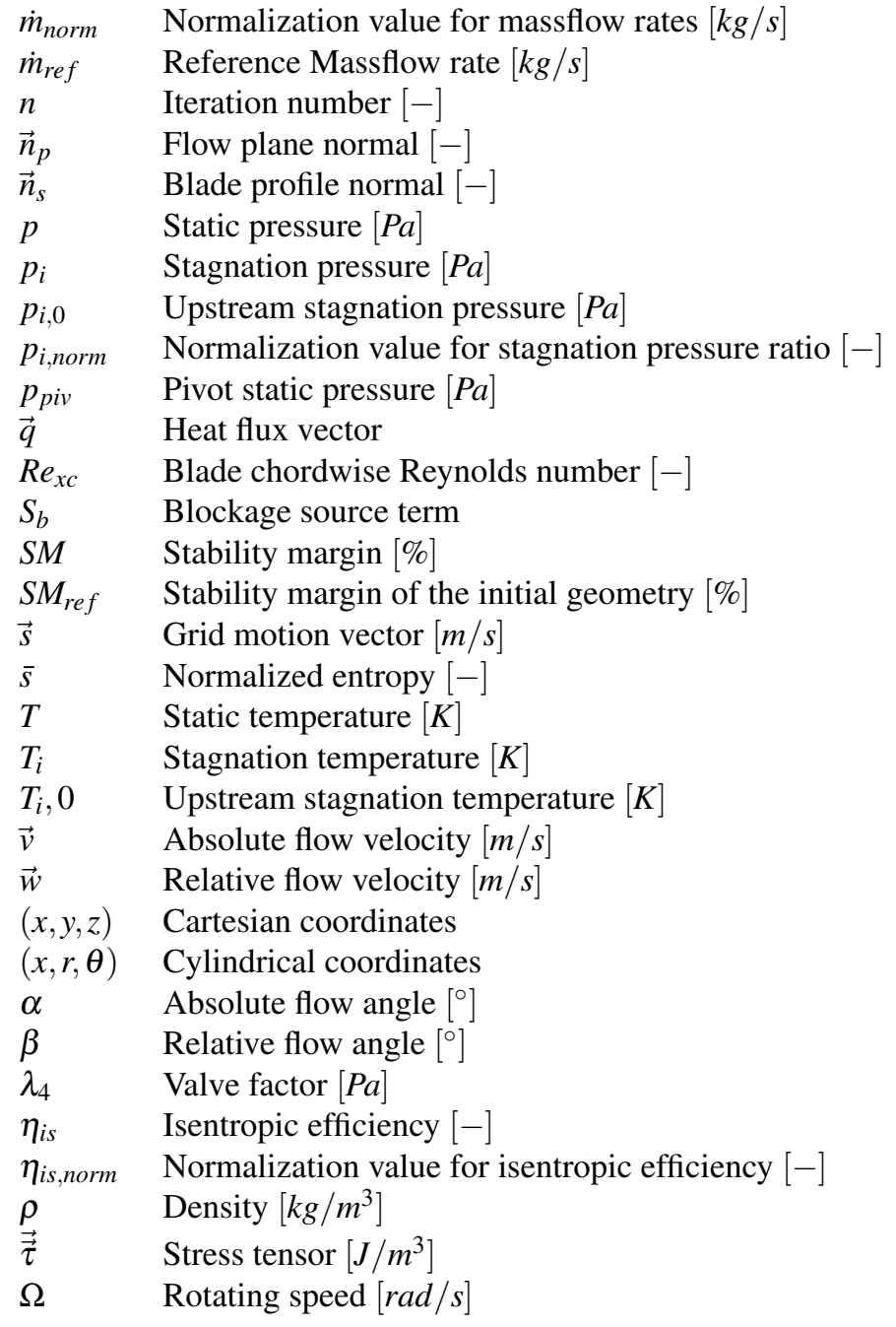

\section{INTRODUCTION}

Many new concepts of civil aircraft involve the issue of propulsive system integration. It notably concerns Ultra High Bypass Ratio (UHBR) turbofan set with:

(i) short inlet $[1,2]$. In this case, it is important to assess whether the profit in terms of load and drag enabled by the slimmer and shorter inlet is not offset by a loss in terms of fan performance and/or operability. Indeed, when subject to specific flight conditions (ex. high angle of attack [3] or cross-wind $[4,5]$ ), the inflow is more inclined to separate, which can result with an incoming heterogeneous flow pattern i.e. an inlet distortion. It is therefore important to assess the fan robustness regarding these kinds of inlet distortion. Nevertheless, most of the flight envelop remains $\left(\right.$ almost $^{1}$ ) absent of distortion. The problem of airframe-

\footnotetext{
${ }^{1}$ Angle or Mach number distortions can still remain because of the nacelle face droop angle and thus negatively affect the fan performances.
}

engine interaction can however concern the whole flight envelop when considering innovating engine integration such as

(ii) Boundary Layer Ingesting (BLI) propulsive systems [6,7, $8,9,10]$. In this kind of configuration, the fan operates constantly within the boundary layer of the fuselage.

Whatever the case, inlet-fan interactions cannot be anymore omitted from the intake or fan design process. It is therefore important to take them effectively and efficiently into account, even during the first steps of the design.

The impact of inlet distortion has been assessed in many studies $[11,12,13]$. They have in particular emphasized the importance of not only stagnation pressure [14] but also swirl distortion to account for performance and stability margin losses and have proposed relevant metrics to assess these distortions $[15,16]$.

In order to mitigate the drop of efficiency and stability margin, several authors have addressed the issue of fan stage design in distorted conditions. In his PhD thesis, Hall [17] has explored the impact of loading distribution variations in the propagation of distortion indicators and diffusion factors. This large parametric study has been possible by means of a low fidelity CFD methodology involving body-force source terms in order to account for the fan and OGV load and deviation effects. Among many conclusions, the author indicates that non-axisymmetric stator design is a promising solution in order to improve the fan stage efficiency. Gunn et Hall $[18,19]$ confirmed this indication with experimental validations. Incidentally, they demonstrated the ability of URANS computations to effectively reproduce the fan stage behavior in their applied distorted conditions. The NASA BLI subscale high-bypass ratio fan stage tested at the Glenn Research Center [20] is perhaps the most advanced research attempt to assess a robust design in BLI conditions, not only in terms of aerodynamic performances [21] but also in terms of aeromechanical response [22].

The previously mentioned papers involve expensive experimental campaign or high fidelity URANS computations. However, design iterations require more efficient methodologies while still having to account for the performance metric sensitivities to geometric variations. To this end, body-force methods represent an interesting way of reducing computational cost. Several models have already shown promising results not only to reproduce potential fan stage effects on inlet aerodynamics $[1,23,2,24]$ but also to describe fan working conditions [25,26,27], notably in distorted conditions $[17,28]$.

In order to address the problem of effectively and efficiently conducting a fan design in distorted conditions, the objectives of this study are to :

(i) assess the extend to which the body-force method can reproduce both fan working conditions and design parameter sensibilities when subject to inlet distortion and to 
(ii) test its ability to be used in an automated optimization process.

After a brief description of the test-case, the body-force formulation and the numerical set-up, an assessment of the body-force methodology is presented. This validation is based on comparisons with RANS and URANS computations. Then, the optimization process based on an arbitrary large design domain is presented. Results are finally discussed and conclusions are drawn so as to stress what must be improved for the methodology to be used in an industrial environment.

\section{TEST-CASE AND NUMERICAL SET-UP \\ General information}

The selected test-case is a Safran Aircraft Engines fan stage scale model representative of UHBR developments. In particular, the fan stage targets very high bypass and low stagnation pressure ratios as presented in TABLE 1 .

TABLE 1. Fan Stage Characteristics

\begin{tabular}{cc}
\hline \hline Bypass ratio [-] & $\approx 16$ \\
\hline Nominal pressure ratio [-] & $<1.4$ \\
\hline \hline
\end{tabular}

\section{Body-force modeling}

The body-force approach consists in replacing the bladed region by an axisymmetric volume where blockage, momentum and energy source terms are applied in each cell. Source terms are a combination of external data and flow proprieties so as to reproduce the effects of the blades. The equation system (1) presents these source terms applied in the right-hand side of the simplified Navier-Stokes equations.

$$
\begin{cases}\frac{\partial \rho}{\partial t}+\operatorname{div}[\rho(\vec{v}-\vec{s})] & =S_{b}=\frac{\rho \vec{v}}{b} \cdot \operatorname{grad}(b) \\ \frac{\partial \rho \vec{v}}{\partial t}+\operatorname{div}[\rho \vec{v} \otimes(\vec{v}-\vec{s})+p \overrightarrow{\vec{I}}-\overrightarrow{\vec{\tau}}] & =S_{b} \vec{v}+\rho \vec{f} \\ \frac{\partial \rho e_{t}}{\partial t}+\operatorname{div}\left[\rho e_{t}(\vec{v}-\vec{s})+p \vec{v}-\overrightarrow{\vec{\tau}} \cdot \vec{v}+\vec{q}\right] & =S_{b} h_{t}+\rho r \Omega f_{\theta}\end{cases}
$$

In particular, $S_{b}=\frac{\rho \vec{v}}{b} \cdot \operatorname{grad}(b)$ serves to model blockage effects and depends on the blockage factor $b=e_{x} / H$ which is the ratio between the blade axial thickness $e_{x}$ and the blade to blade staggered spacing $H$ [29]. It is then all about finding suitable values for the body-forces $\vec{f}$. In this study, the method follows a formulation based on a decomposition of the body-forces $\vec{f}$ within the flow plane ${ }^{2}$ as illustrated by FIGURE 1 . In this particular

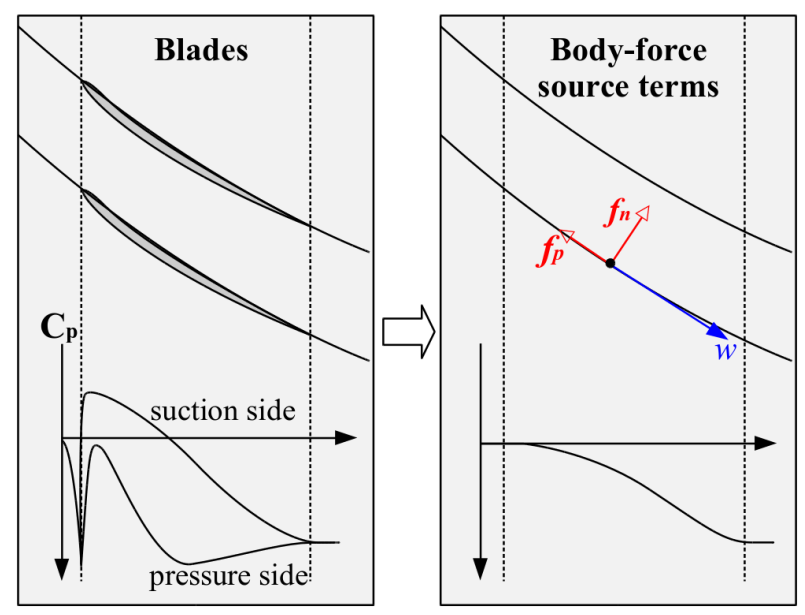

FIGURE 1. Body-force principle and decomposition within the flow plane [30]

plane, the body-forces can be decomposed in only two components $\vec{f}=\vec{f}_{n}+\vec{f}_{p}$. The first component $\vec{f}_{n}$ is perpendicular to the flow relative velocity $\vec{w}$ and serves to reproduce main deviation and loading effects. The second component $\vec{f}_{p}$ is parallel to $\vec{w}$ and serves to add losses as directly proportional to entropy gradients [31]. The selected formulation is described by equations (2) and (3) $[17,2]$ :

$$
\begin{gathered}
f_{n}=\frac{w^{2}}{2 b H} 2 \pi \delta \times \underbrace{\left\{\begin{array}{l}
\frac{1}{\sqrt{1-M_{r e l}}} \text { if } M_{r e l}<1 \\
\frac{4}{2 \pi \sqrt{M_{r e l}{ }^{2}-1}} \text { if } M_{r e l}>1
\end{array}\right.}_{\text {Compressible effects }} \\
f_{p}=\frac{w^{2}}{2 b H}\left[K_{p 0}+2 \pi\left(\delta-\delta_{0}\right)^{2}\right]
\end{gathered}
$$

in which:

(i) $M_{r e l}(x, r, \theta)$ is the relative Mach Number,

(ii) $K_{p 0}=2 \times 0.0592 \times R e_{x c}^{-0.2}$ is the friction drag coefficient and depends on the local Reynolds number $R e_{x c}$ (which is based on the chordwise position $x_{c}$ of the blade) and

(iii) $\delta=\arcsin \left(\frac{\vec{w} \cdot \vec{n}_{s}}{\|\vec{w}\|}\right)$ is the local deviation in the plane defined by the blade profile normal $\vec{n}_{s}(x, r)$.

\footnotetext{
${ }^{2}$ The normal $\vec{n}_{p}$ of the flow plane is defined by $\left(\vec{f} \cdot \vec{n}_{p}=0\right)$
} 
(iv) Also, $\delta_{0}(x, r)$ is the deviation extracted from a body-force computation at maximum efficiency where only friction losses are applied i.e. $f_{p}=w^{2} K_{p 0} /(2 b H)$.

This model is particularly easy to implement as it exclusively depends on the blade geometry as well as a single calibration parameter $\delta_{0}$, the establishing of the latter "only" requiring the massflow rate value at maximum efficiency. Moreover, this model has already shown promising results $[17,2]$. However, one of the major noted limitation is the inability of the model to accurately reproduce stall behavior at low massflow rate.

\section{Numerical set-up}

CFD simulations are performed with the elsA solver developed by Safran, Airbus and ONERA on structured meshes [32]. Equations are discretized in space using a cell-centered finite volume method with the second order upwind scheme of Roe [33]. The $k-l$ model of Smith [34] ensures turbulence closure. Steady simulations are run with an implicit pseudo-time marching method with backward Euler scheme. Unsteady simulations use a Gear scheme for integration in time with 15 subiterations process. Two computational domains are considered. The first one refers to meshed blades (U)RANS computations and is presented in FIGURE 2. The grid was generated using NUMECA

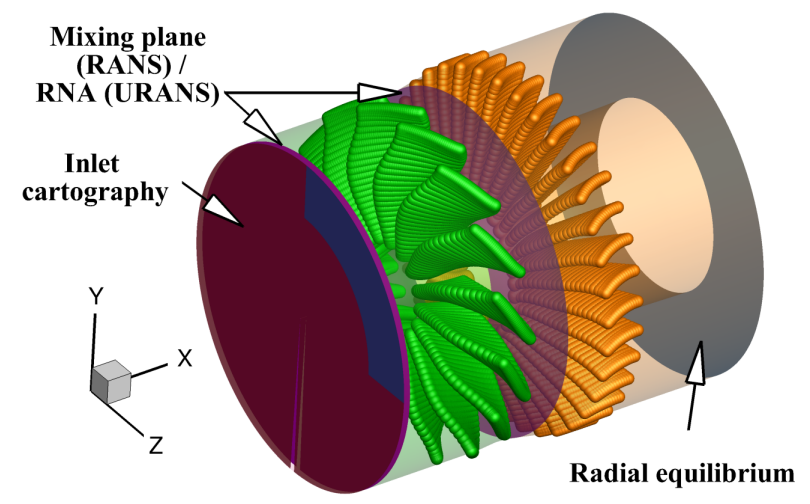

FIGURE 2. URANS Computational Domain

AUTOGRID ${ }^{T M}$ software [35] and is relatively coarse with a domain mesh size of approximately $60 \times 10^{6}$ elements $\left(2.5 \times 10^{6}\right.$ elements for periodic sector RANS computations). The second domain presented in FIGURE 3 is used for body-force RANS computations. The grid was generated using the Cassiopee software [36] with a domain mesh size of approximately $4.0 \times 10^{6} \mathrm{el}-$ ements. Sectors of stagnation pressure losses are applied as inlet conditions. The inlet cartography applied is a constant stagnation pressure drop of $\Delta p_{i} / p_{i, 0}=10 \%$ applied in the last $\Delta h=35 \%$ of

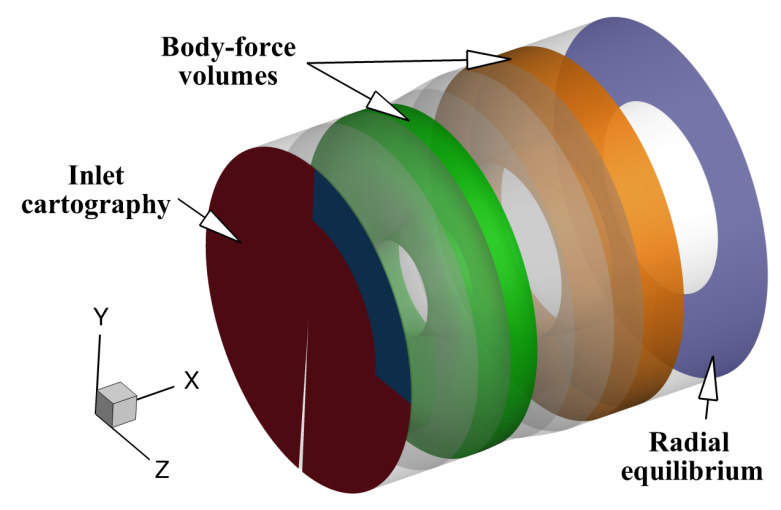

FIGURE 3. Body-force Computational Domain

the span and over a circumferential sector of $\Delta \theta=90^{\circ}$ (cf. FIGURE 2 and FIGURE 3 ). The outflow boundary conditions are set with a radial equilibrium defined by a pivot static pressure $p_{p i v}$ located at the hub. Iso-rotating speed characteristics are obtained by adjusting the pivot static pressure through a valve factor $\lambda_{4}$ :

$$
p_{p i v, n+1}=p_{r e f}+\lambda_{4}\left(\frac{\dot{m}_{n}}{\dot{m}_{r e f}}\right)^{2}
$$

where $\dot{m}_{n}$ is the massflow rate at iteration $n$ and $\dot{m}_{\text {ref }}$ and $p_{\text {ref }}$ are massflow rate and static pressure values of reference.

\section{BODY-FORCE MODELING ASSESSMENT}

In order to validate the body-force modeling formulation used in this study, fan stage simulations have been run in both isolated and distorted inlet conditions. The objectives are to assess the ability of the body-force methodology to account for:

(i) sensitivities of design parameters and

(ii) fan working behavior in distorted conditions.

Sensitivities of fan design parameters have been assessed in clean inflow conditions. Selected parameters are respectively the leading and trailing edges metal angles $\beta_{s q, 1}$ and $\beta_{s q, 2}$ and the chord length $c$ (cf. FIGURE 4 for illustration). Design variations have firstly been applied uniformly throughout the blade span. The relative blade thickness as well as centers of gravity have been conserved through the process. FIGURES 5, 6 and 7 present iso-speed fan characteristic comparisons between the "meshed blades" and the "body-force" RANS computations respectively for variations of $\Delta \beta_{s q, 1}=+/-2^{\circ}, \Delta \beta_{s q, 2}=+/-2^{\circ}$ and $\Delta c / c=+/-5 \%$. The stagnation pressure ratio $p_{i} / p_{i, 0}$ and 


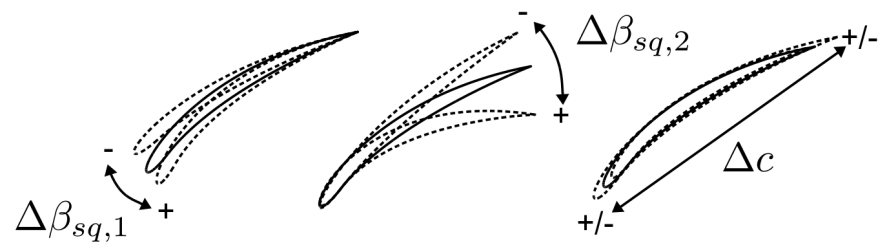

FIGURE 4. Design Parameters Variations
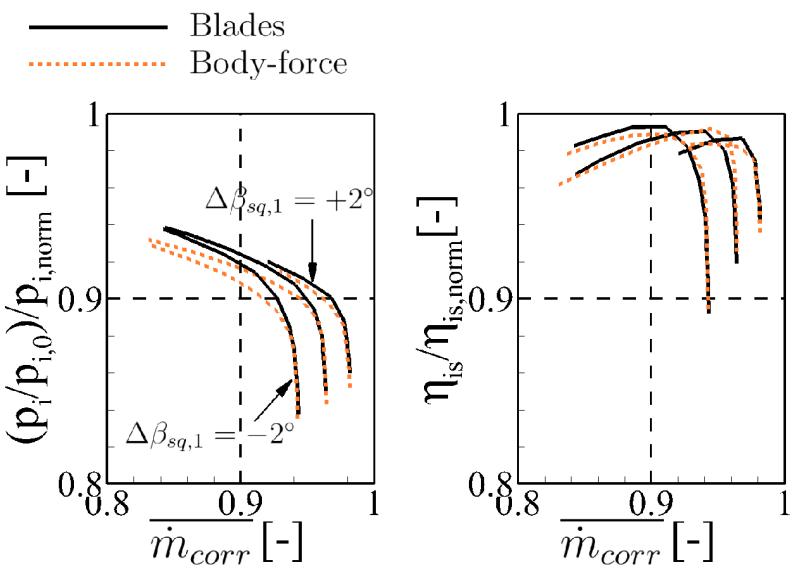

FIGURE 5. Stagnation Pressure Ratio and Isentropic Efficiency Characteristics for Blade and Body-force Computations - Variations of $\beta_{s q, 1}$
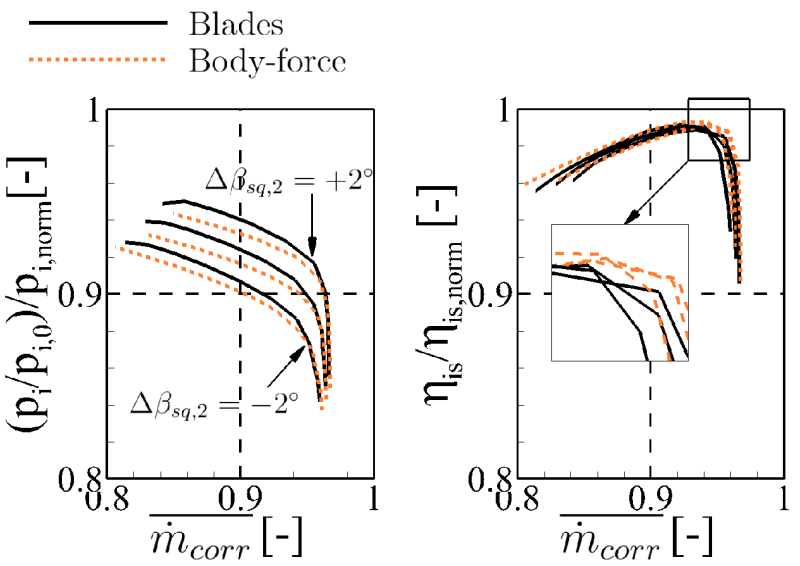

FIGURE 6. Stagnation Pressure Ratio and Isentropic Efficiency Characteristics for Blade and Body-force Computations - Variations of $\beta_{s q, 2}$

the isentropic efficiency :

$$
\eta_{i s}=\frac{\left(p_{i} / p_{i, 0}\right)^{\frac{\gamma-1}{\gamma}}-1}{T_{i} / T_{i, 0}-1}
$$

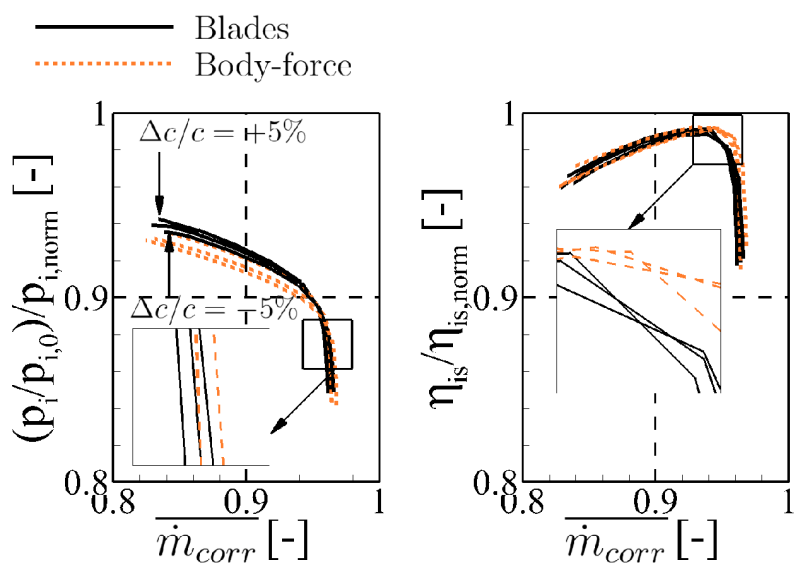

FIGURE 7. Stagnation Pressure Ratio and Isentropic Efficiency Characteristics for Blade and Body-force Computations - Variations of $c$

have been normalized (via the values $p_{i, \text { norm }}$ and $\eta_{i s, n o r m}$ ) and are plotted against the normalized corrected massflow rate $\overline{\dot{m}_{\text {corr }}}$ defined by equation (6):

$$
\overline{\dot{m}_{\text {corr }}}=\frac{\dot{m} \frac{\sqrt{T_{i, 0} / T_{i, r e f}}}{p_{i, 0} / p_{i, r e f}}}{\dot{m}_{\text {norm }}}
$$

where $p_{i, 0}$ and $T_{i, 0}$ are the stagnation pressure and temperature taken upstream while $p_{i, r e f}=101325 \mathrm{~Pa}$ and $T_{i, \text { ref }}=288.15 \mathrm{~K}$ are standard values at ISA-0. Also, $\dot{m}$ is the massflow rate and $\dot{m}_{\text {norm }}$ is a normalization value. Globally, conclusions are the same for the three studied parameters. Sensitivities are relatively well reproduced. Yet, some notable differences can be highlighted. First, the body-force formulation underestimates the stagnation temperature ratio. As it also underestimates the stagnation pressure ratio, errors balance each other out and the isentropic efficiency is well accounted for. This underestimation of stagnation temperature ratio can be explained by the change of flow behavior along the span i.e. subsonic near the hub and transonic near the shroud, which is not accounted for by the body-force model. Profiles in the transonic part of the blade span denote an absence (or even a change of sign) of camber approximately in the fist $60 \%$ of chordwise coordinate, which is typical of transonic fan blade design. Figure 8 presents the evolution of the circumferentially averaged and normalized tangential force $\bar{f}_{\theta}$ of the fan against the normalized axial chord length $\bar{c}_{x}$ for both the body-force and RANS computations taken at maximum efficiency. It notably shows that at $h=20 \%, \bar{f}_{\theta}$ behaves similarly in both cases. Indeed, at this relative height (and especially at maximum efficiency), the flow that crosses the fan remains subsonic and therefore the assumption that the flow deviation follows the blade profile is well respected. Therefore, 


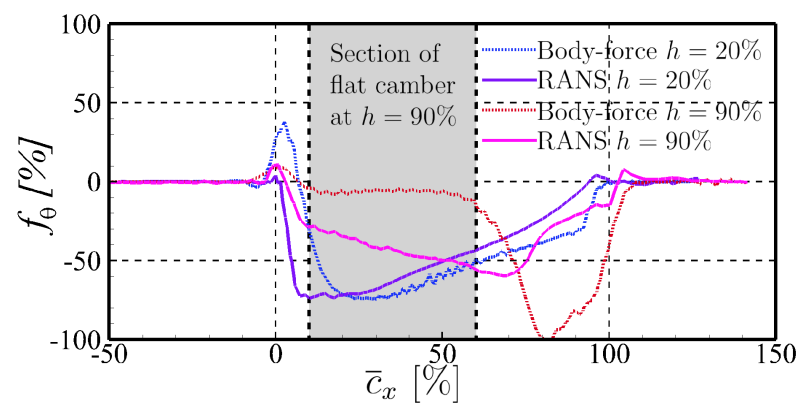

FIGURE 8. Circumferentially averaged tangential force $\bar{f}_{\theta}$ comparison at $h=20 \%$ (subsonic behavior) and $h=90 \%$ (transonic behavior) of the body-force model and RANS (meshed blades) results

the formulation (2) which assumes a proportionality between the normal force applied $f_{n}$ and the local deviation $\delta$ manages well to reproduce the flow behavior in this region. However, when a shock occurs within the blade-to-blade channel, additional deviation is generated because of a decrease of the flow relative velocity component normal to the shock front. Thus, it locally implies additional loads which are not reproduced by the bodyforce model. This can be observed in Figure 8 by noting that tangential forces $\bar{f}_{\theta}$ at $h=90 \%$ are drastically different. While the body-force model induces a tangential force only in the region where the blade camber is not close to zero (which is the case outside of the grey area plotted in Figure 8), deviations occur alongside the entire channel in the RANS computations due to the effect of the shock. Eventually, the integrated tangential forces applied to the flow and therefore the stagnation temperature ratio differ between the two approaches. Besides, it must be noted that maximum efficiency values evolution is not always conserved. It is notably the case for variation of $\Delta \beta_{s q, 1}$. The maximum isentropic efficiency slightly decreases when $\Delta \beta_{s q, 1}$ increases for the "meshed blades" computations while the maximum efficiency values are lower than the reference for both applied variations for the "body-force" computations (cf. FIGURE 5 right caption). Nevertheless, the body-force methodology manages well to reproduce the massflow rate blockage values predicted by the meshed blades computations.

A first assessment in distorted conditions has been performed on the reference geometry. In this kind of configuration (cf. FIGURE 2 and FIGURE 3), assuming that the fan stage working conditions remain axisymetric is not defendable anymore. Therefore, it is necessary to assess variations of working conditions throughout the circumference. In order to properly associate upstream and downstream conditions, a specific postprocessing, firstly presented by Fidalgo et al. (2012) [14], has been applied. It consists in dividing a reference section upstream of the fan leading edge into multiple sectors. Then, for each upstream sector, an associated sector located downstream of the fan trailing edge is identified using the intersections between the streamsurfaces generated from the upstream sector boundaries and the downstream section. Upstream and downstream sections have been selected at axial locations close to the fan leading and trailing edges. FIGURE 9 shows an example of the sectors result-

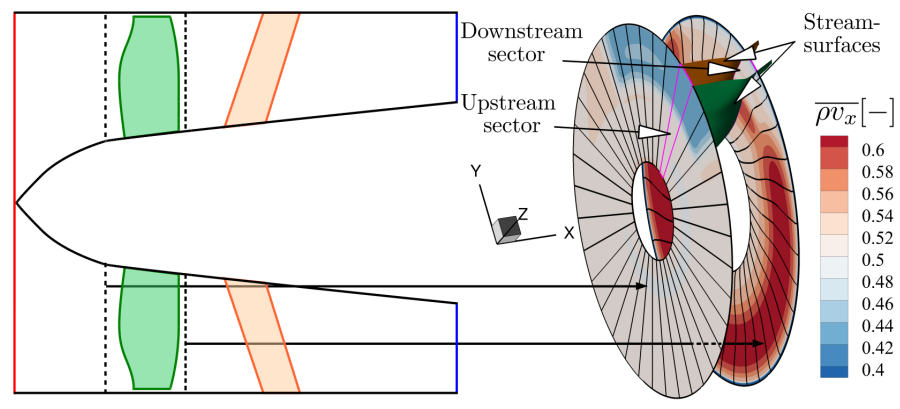

FIGURE 9. Post-processing in Distorted Inlet Conditions

ing from this post-processing. Compared to Blade URANS computations, using body-force modeling methods greatly simplifies this kind of post-processing by providing flow field directly in the absolute frame. Therefore, there is no need for complex averaging process to translate results from the rotating to the nonrotating absolute frame. Averaging the flow solution within the identified sectors eventually enables to plot the evolution of the fan working conditions throughout an entire revolution, which is represented by an orbit in the performance map. In the study, the upstream section has been divided between 96 sectors. This quantity results from a convergence study on orbit shapes and stability margins. FIGURE 10 presents the performance map in which both blade and body-force simulations are compared. Stagnation pressure orbits of both Blade URANS and body-force RANS computations are in good agreement. It demonstrates the ability of the body-force model to reproduce fan working conditions in distorted area in terms of stagnation pressure ratio. In particular, the closest local working condition $M$ to the stability limit $S^{3}$ is well captured. This enables an efficient assessment of the stability margin defined in this study by equation (7):

$$
S M=\frac{\left(\frac{p_{i} / p_{i, 0}}{\dot{m}_{c o r r}}\right)_{S}-\left(\frac{p_{i} / p_{i, 0}}{\dot{m}_{c o r r}}\right)_{M}}{\left(\frac{p_{i} / p_{i, 0}}{\dot{m}_{\text {corr }}}\right)_{M}}
$$

However, larger differences arise with the prediction of the isentropic efficiency. This is mainly attributed to the previously emphasized fact that the body-force formulation used in this study

\footnotetext{
${ }^{3}$ The stability limit is defined by the point of maximum static pressure ratio of the characteristic (i.e. $\left.\left(p / p_{i, 0}\right)_{\max }\right)$ [37]
} 

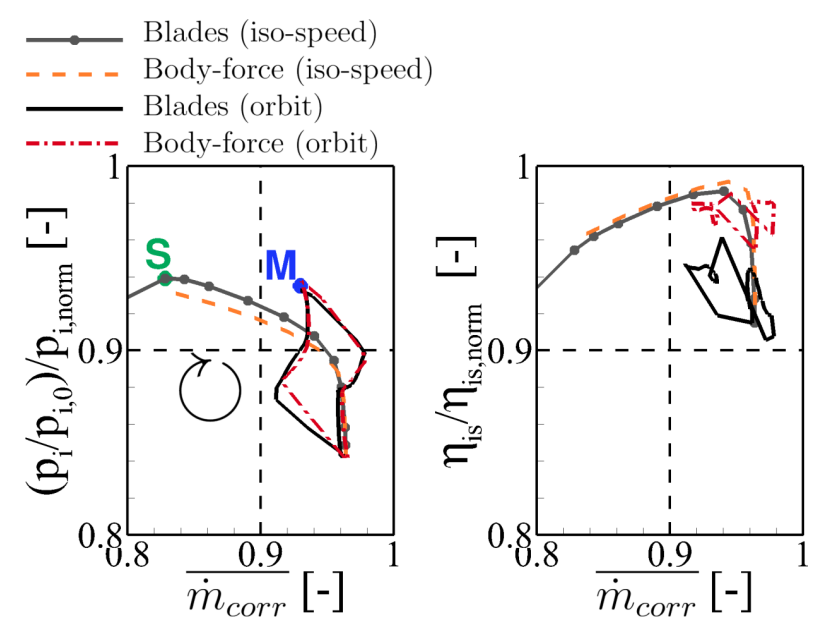

FIGURE 10. Stagnation Pressure Ratio and Isentropic Efficiency Characteristics and Orbits in Distorted Conditions for Blade and Bodyforce Computations

tends to underestimate the stagnation temperature rise across the fan. As a result, it leads to an overestimation of the isentropic efficiency.

Concerning the distortion transfer, FIGURE 11 presents a
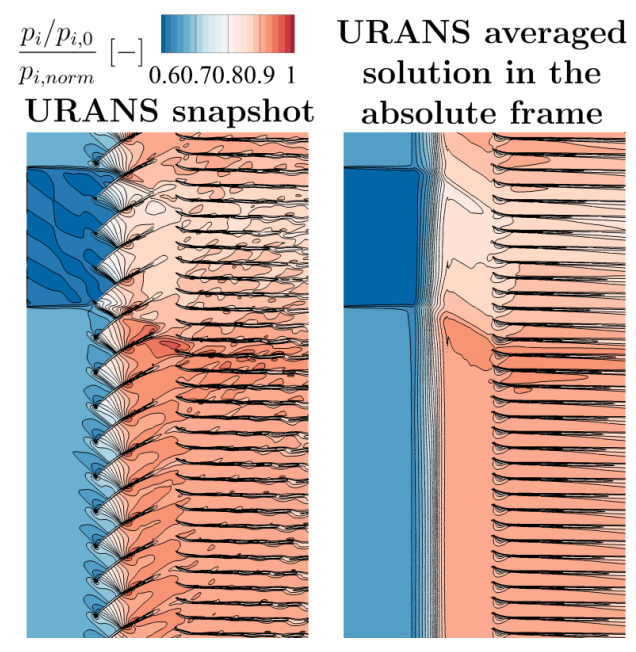

\section{Body-force computation}

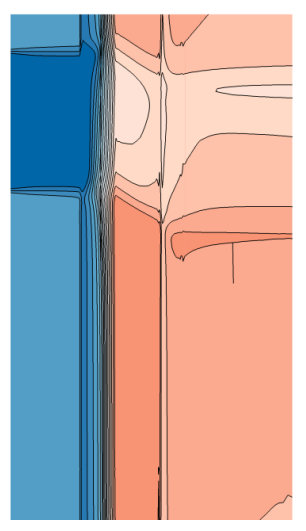

FIGURE 11. Slices at $h=85 \%$ colored with stagnation pressure ratio. Comparisons of URANS (snapshot and absolute frame time averaged solution) and body-force methodologies

comparison of slices colored by stagnation pressure ratio between an URANS solution snapshot, its time-average (in the nonrotating frame) and a body-force solution. Results are qualitatively in good agreement. In addition, FIGURE 12 shows fluctu-
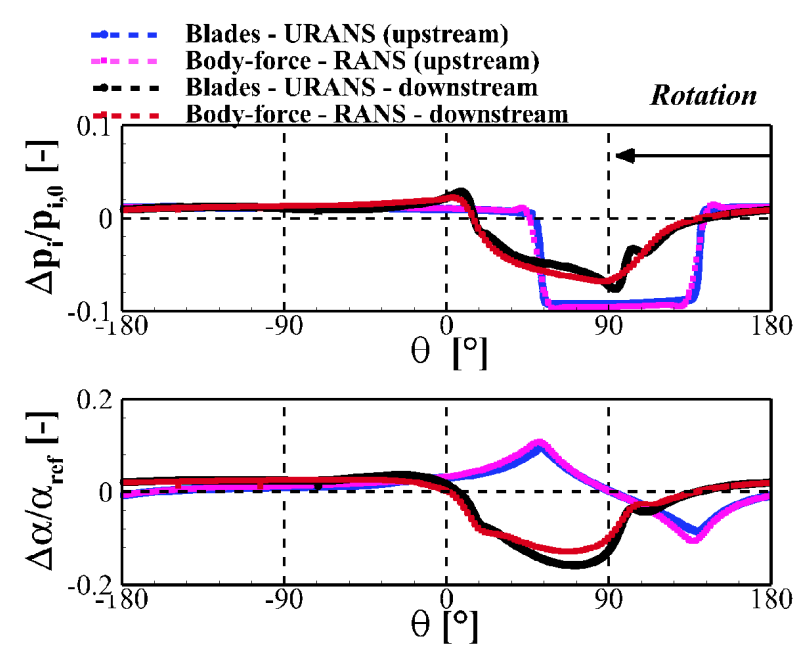

FIGURE 12. Flow fluctuations throughout the circumference upstream and downstream of the fan at $h=83 \%$. Body-force and URANS comparison

ations in terms of stagnation pressure and swirl ${ }^{4}$ as functions of the circumferential coordinate $\theta$ at $h=83 \%$. Again, it emphasizes the fact that the body-force method manages well to reproduce the distortion transfer across the fan row. Nevertheless, it is possible to note that the body-force model underestimates the swirl variation downstream of the fan. This once more highlights the underestimation of the fan work predicted by the body-force model compared to (U)RANS computations.

Altogether, the body-force modeling formulation, although not perfect, has been reckoned reliable enough to assess fan working conditions when subject to inlet distortion. In particular, this methodology has been used in order to efficiently explore a design domain so as to assess the impact of geometric parameters on fan efficiency and stability margin in distorted conditions. The description and the results of this study are discussed in the next section.

\section{APPLICATION FOR DESIGN OPTIMIZATION IN DIS- TORTED CONDITIONS}

The design domain has been arbitrary chosen as such : $\Delta \beta_{s q, 1} \in\left[-2^{\circ}, 2^{\circ}\right], \Delta \beta_{s q, 2} \in\left[-2^{\circ}, 2^{\circ}\right]$ and $\Delta c \in[-5 \%, 5 \%]$ at two control relative heights $h \in\{50 \%, 100 \%\}$. Six design parameters are therefore considered and only concern the fan geometry.

One of the challenges of this study is to correctly identify the stability margin for each assessed points. Indeed, it has been emphasized that the body-force formulation does not reproduce

\footnotetext{
${ }^{4}$ In the case of swirl fluctuations, the value of reference $\alpha_{\text {ref }}$ has been taken differently downstream and upstream so as to keep the fluctuations within similar amplitude range.
} 
the fan behavior outside of the stability limit i.e. the positive slope of the characteristic. Therefore, it is necessary to determine these instability limits for each new design. This is quite inconvenient as it a priori requires to compute a new characteristic with "blades" RANS computations for each evaluated design. In order to reduce the computational cost, it has been decided to indeed compute these characteristics but only at the domain border, which still implies $2^{6}=64$ designs. Then, the stability limit conditions have been estimated via an interpolation by means of Radial Basis Functions (RBF) over these border values. FIGURE 13 presents the computed characteristics (left caption)

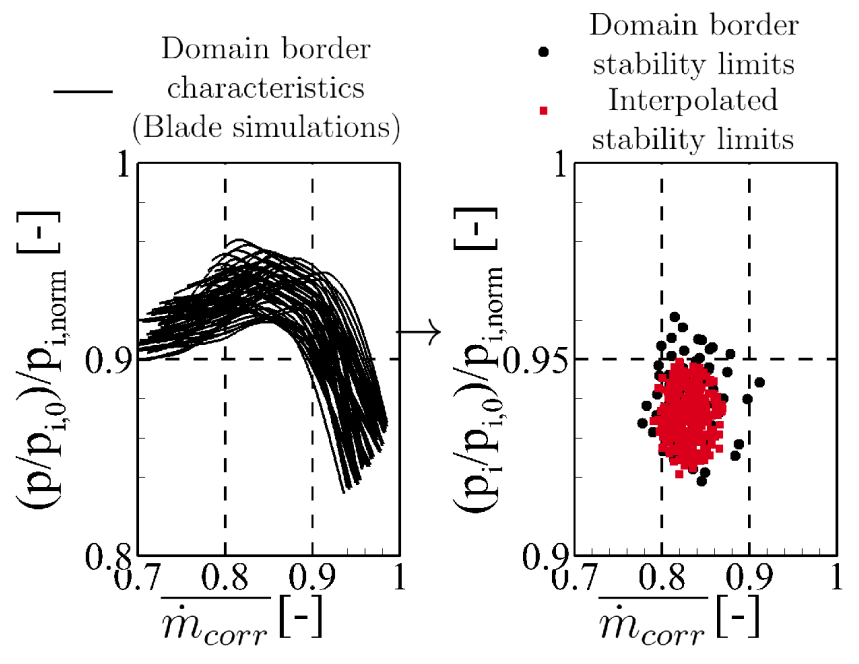

FIGURE 13. Domain border characteristics and interpolated stability limits

with the associated stability limits (right caption) and those of 320 designs interpolated from the border values. These designs have been defined by a Latin Hypercube Sampling (LHS) which enables to efficiently explore the domain (i.e. with few assessed points) [38]. Then, each design has been assessed by a bodyforce computation in the same distorted conditions. Design variations can modify the current massflow rate and stagnation pressure ratio. Therefore, comparisons are made at different working conditions, which make the whole exercise easily questionable. However, a same valve factor $\lambda_{4}$ (cf. equation (4)) has been applied for all computations. This enables to locate the working conditions at a relatively same (close) distance from their maximum efficiency reference. Therefore, this study must be understood as rather an assessment of how a given design (at its own defined working condition) is impacted by a distortion than an optimization focus on a single set of specifications. FIGURE 14 and FIGURE 15 present the impacts of the different design parameters respectively in terms of stability margin $\Delta S M$ and isen-
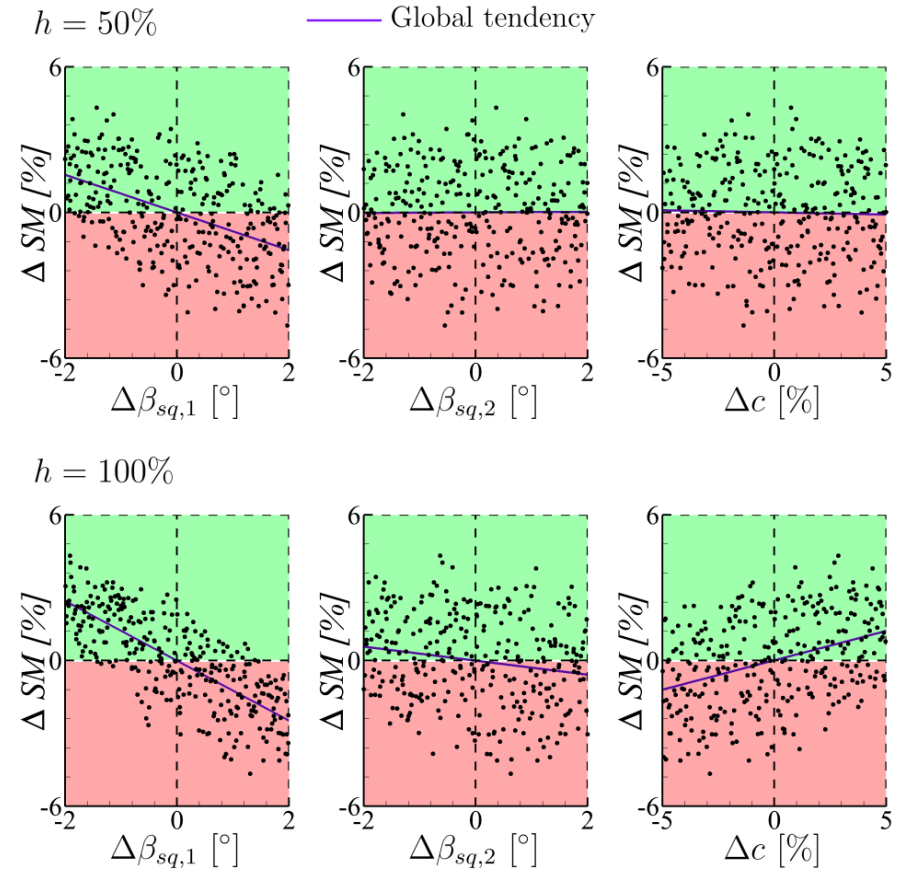

FIGURE 14. Stability Margin Variations against the Design Variables

tropic efficiency $\Delta \eta_{i s}$. These objective functions $\Delta S M$ and $\Delta \eta_{i s}$ are differences between the assessed geometry and the reference design both in distorted conditions. Thus, a positive value means a more robust design than the reference. In FIGURE 14 and FIGURE 15, global tendency slopes have been added to the graphs. These global tendencies are defined so as to minimize the linear regression error. Several comments can be made regarding those results:

(i) The stability margin is improved by increasing the chord length at the tip ( $h=100 \%$ ) (cf. FIGURE 14 bottom-right caption). This tendency makes sense to the extent that the section subject to lower massflow rate (because of the distortion) is located in the last $35 \%$ of relative height. Hence it is the most critical sector regarding stability. Thus, increasing locally the solidity in this region (via an increase of chord length) can improve the stability margin. However, it is not always possible to use this lever because of structural considerations.

(ii) Decreasing the trailing edge metal angle $\beta_{s q, 2}$ enables to improve both stability and efficiency. This is not surprising as it also decreases the global fan work.

(iii) Finally, leading edge metal angle variations $\Delta \beta_{s q, 1}$ have an antagonist effect on the stability margin and the isentropic efficiency. Also, the fact that this parameter has more impact on the isentropic efficiency in the lower part of the relative height $(h=50 \%)$ can be explained as a higher propor- 

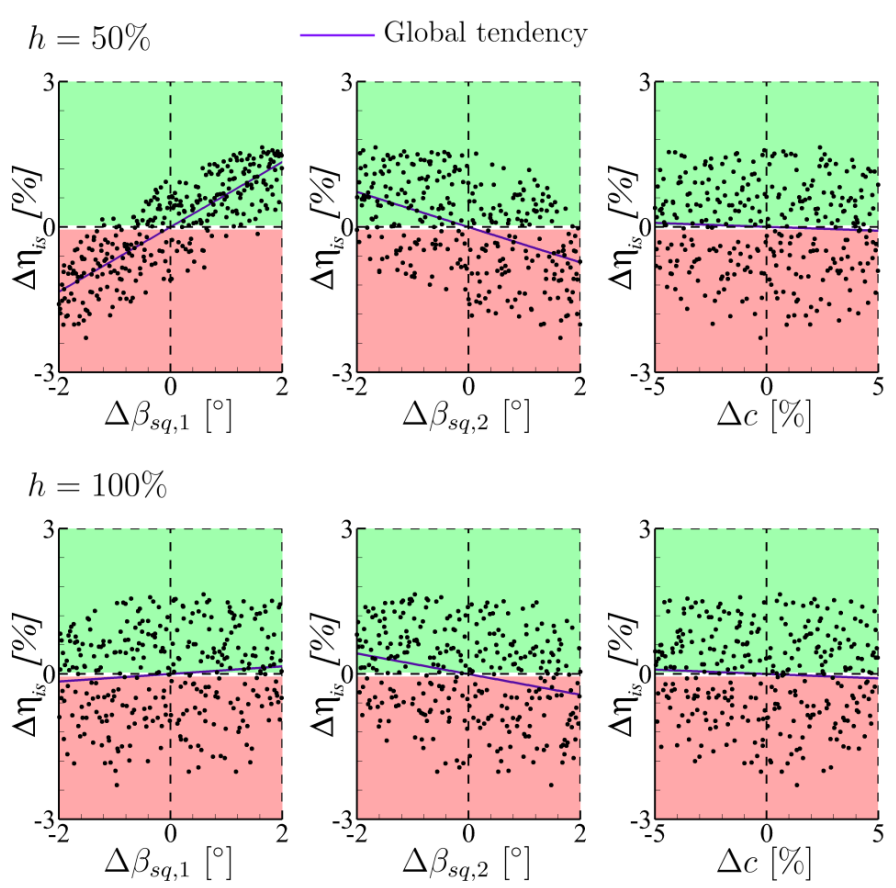

FIGURE 15. Isentropic Efficiency Variations against the Design Variables

tion of the massflow rate gets through this region. Besides, the fact that an increase of $\beta_{s q, 1}$ applied in the distorted region improves the isentropic efficiency can be easily explained as profiles are thus re-aligned with the decelerated flow.

Finally, these previous tendencies could have been a priori guessed by fan designers. However, the methodology provides a quantification of these tendencies. They also highlight the fact that the body-force methodology provides consistent results in spite of its previously highlighted flaws (especially regarding the prevision of the isentropic efficiency).

The results have also been used to build a meta-model by means of RBF. Then, a Non-dominated Sorting GA 2 (NSGA2) [39] algorithm based on this meta-model has enabled to determine an optimized Pareto front represented in FIGURE 16. The meta-model mean and maximal errors are respectively $0.06 \%$ and $0.30 \%$ for the stability margin assessment and $0.03 \%$ and $0.11 \%$ for the isentropic efficiency ${ }^{5}$.

From the computed Pareto front, three candidates have been picked-up so as to illustrate geometric and performance changes on relevant designs (cf. large colored points in FIGURE 16). A

\footnotetext{
${ }^{5}$ These errors are calculated by building a meta-model based on a domain which is relieved of a single point. The relative difference between the computed and the assessed values defines the local error and this process is then repeated for every computed point.
}

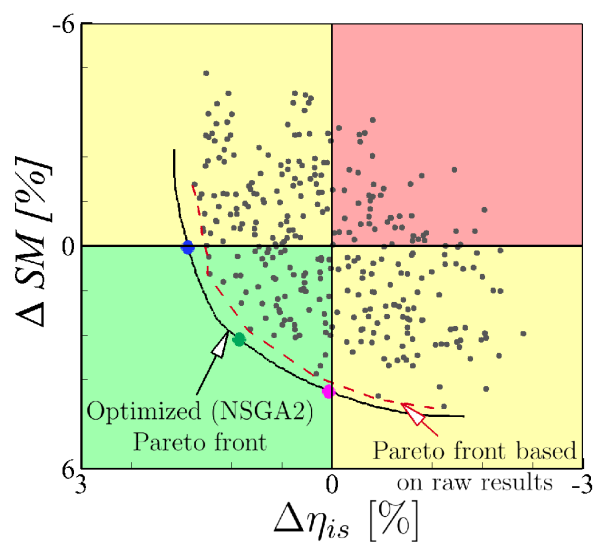

FIGURE 16. Optimized and Raw Pareto Fronts

first candidate has been selected in order to maximize the stability margin $\left(\Delta S M=+3.9 \%, \Delta \eta_{i s}=+0.0 \%\right)$ without decrease of the initial isentropic efficiency (pink point of FIGURE 16)). Oppositely, another one has been selected so as to maximize the isentropic efficiency $\left(\Delta S M=+0.04 \%, \Delta \eta_{i s}=+1.5 \%\right)$ without deterioration of the stability margin (blue point of FIGURE 16)). Finally, a third and last one has been selected as a trade-off $\left(\Delta S M=+2.52 \%, \Delta \eta_{i s}=+1.09 \%\right)$ between the previous ones (green point of FIGURE 16). FIGURE 17 shows the geometric variations in reference to the initial geometry that have been applied to these selected candidates. It shows that the candidate

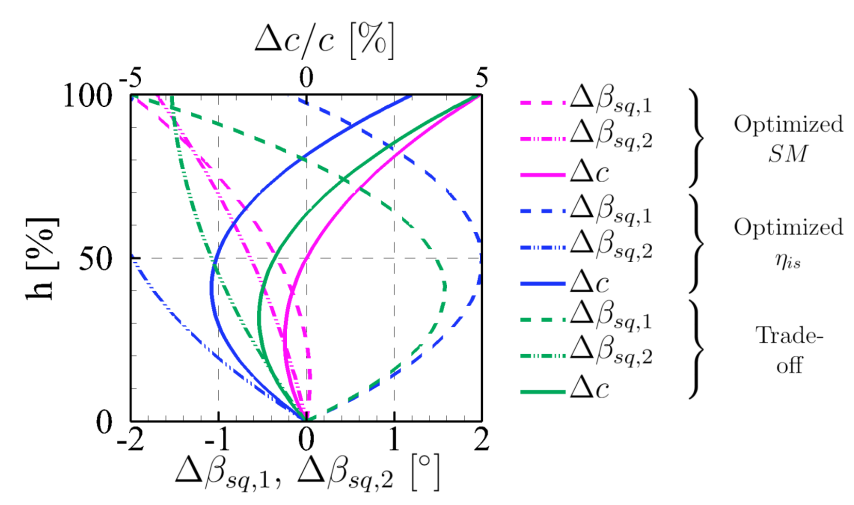

FIGURE 17. Geometric Variations Comparison between the Selected Candidates

that maximizes the stability margin reaches this achievement by a strong increase of chord length $c$ and a decrease of the inlet metal angle $\beta_{s q, 1}$ at high values of relative height $h>0.5$. On the other hand, the candidate that maximizes the isentropic efficiency manages so by a strong increase of $\beta_{s q, 1}$ at mid-height and 
by a global decrease of the outlet metal angle $\beta_{s q, 2}$. The "tradeoff" candidate shows variations somewhere in-between the two others. Moreover, these values are consistent with the global tendencies showed by FIGURE 14 and FIGURE 15. Body-force computations have confirmed the meta-model predicted values. This can be illustrated by FIGURE 18 which shows the orbits of
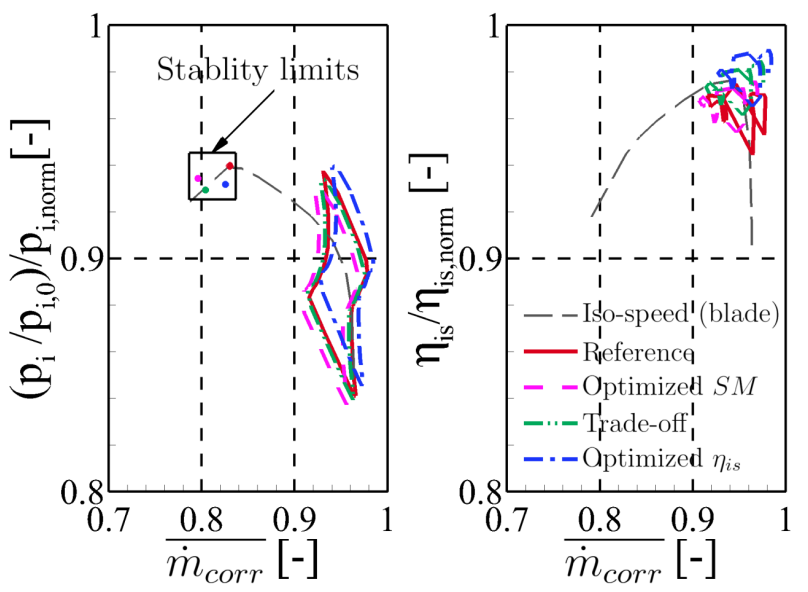

FIGURE 18. Fan Orbits Comparison between the Selected Candidates

circumferential working conditions of these three selected candidates in terms of stagnation pressure ratio $p_{i} / p_{i, 0}$ (left caption) and isentropic efficiency (right caption). Stability limits have been added in the left caption in order to provide a reference for each orbit and thus to correctly assess the margins.

Finally, in order to assess these results, another URANS computation has been performed using the "trade-off" geometry. Table 2 summarizes the principal results extracted from

TABLE 2. "Trade-off” geometry summarized results

\begin{tabular}{ccccc} 
& $\Delta S M[\%]$ & $\Delta \eta_{i s}[\%]$ & $\frac{\Delta\left(p_{i} / p_{i, 0}\right)}{p_{i} / p_{i, 0}}[\%]$ & $\frac{\Delta \dot{m}}{\dot{m}_{\text {ref }}}[\%]$ \\
\hline \hline NSGA2 & +2.52 & +1.09 & - & - \\
\hline URANS & +0.43 & +1.47 & -0.30 & -0.28 \\
\hline \hline
\end{tabular}

both the optimization process and the URANS computation of this "trade-off" geometry. Compared to the NSGA2 prediction, the URANS computation estimates a far lower gain in stability margin $\Delta S M_{\text {tradeoff,URANS }}=+0.43 \%<\Delta S M_{\text {tradeoff }, N S G A 2}=$ $+2.52 \%$ and a higher gain in terms of isentropic efficiency $\Delta \eta_{\text {is,tradeoff }, \text { URANS }}=+1.47 \%>\Delta \eta_{\text {is,tradeoff }, N S G A 2}=+1.09 \%$

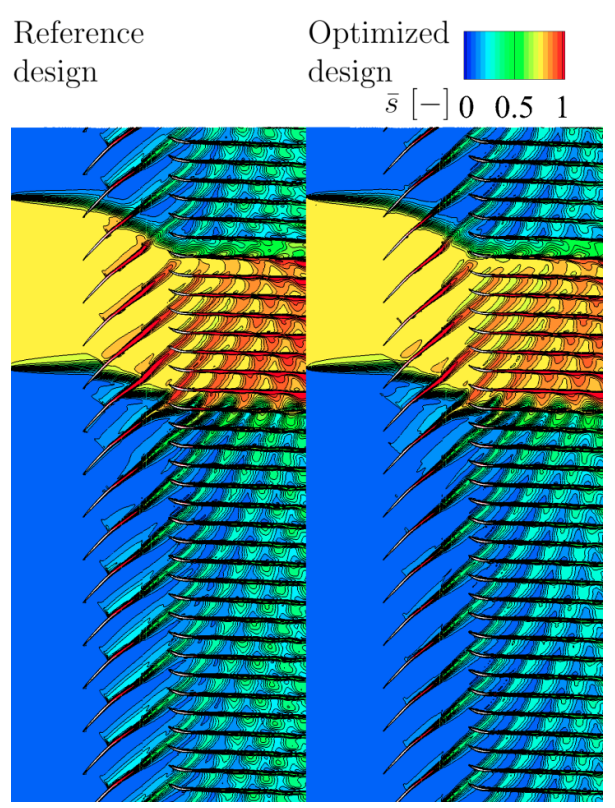

FIGURE 19. Slices at $h=80 \%$ colored by normalized entropy. Comparison between the reference geometry (left) and the "trade-off" optimized design (URANS snapshots)
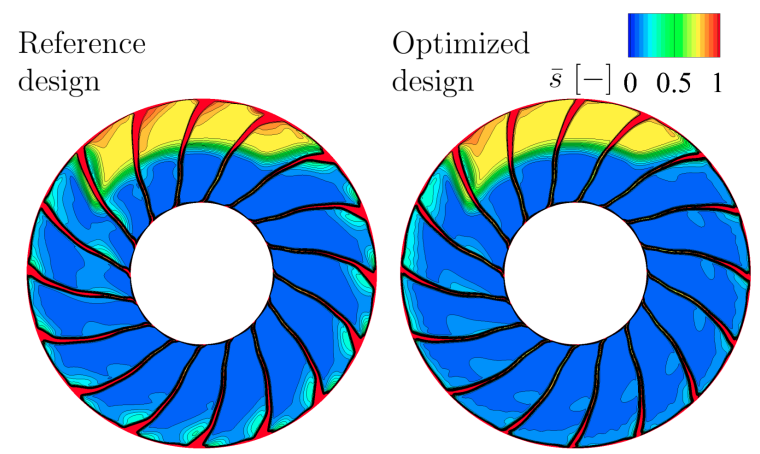

FIGURE 20. Axial station downstream of the fan colored by normalized entropy. Comparison between the reference geometry (left) and the "trade-off" optimized design (URANS snapshots)

while the stagnation pressure ratio remains well conserved $\left(\Delta\left(p_{i} / p_{i, 0}\right) /\left(p_{i} / p_{i, 0}\right)_{\text {ref }}=-0.30 \%\right)$ as well as the massflow rate $\Delta \dot{m} / \dot{m}_{r e f}=-0.28 \%$. Figure 19 and Figure 20 respectively present a comparison of slices at $h=80 \%$ and at a station downstream of the fan colored by the normalized entropy $\bar{s}$. In these figures, it can be qualitatively noted that the optimized geometry produces less entropy than the reference and shows thinner trailing edge wakes, which illustrates the isentropic efficiency gain. Regarding the stability margin prediction, as stagnation pressure orbits are well reproduced by the body-force methodology, one 
would have expected a better match between the predicted and the computed results. Actually, this difference mostly comes from a poor estimation of the stability limit (which is, as a reminder, assessed by RBF interpolation over the extremities of the design domain). Indeed, an underestimation of the massflow rate condition of $5.3 \%$ has been noted compared to the RANS prediction, which calls the entire optimization process into question. Therefore, it underlines the importance of improving the model in order to intrinsically (and thus efficiently) capture the stability limit.

To conclude, even though some discrepancies remain between the computed results and the NASGA2 prediction, tendencies remain correct and the new design happens to be more adapted to the applied inlet distortion.

\section{CONCLUSION, DISCUSSION AND PERSPECTIVES}

In this paper, a body-force approach has been assessed and applied in order to efficiently explore a fan design domain in stagnation pressure distortion conditions. Presented results have highlighted the extend to which the body-force approach can reproduce a fan stage behavior in distorted conditions. Comparisons have been made with both "meshed blades" RANS and URANS computations which require additionnal computational effort by approximately two orders. The study has notably shown that the selected body-force formulation [17,2] globally underestimates the stagnation temperature ratio which leads, in distorted condition, to an overestimation of the isentropic efficiency. However, the global behavior is well reproduced. In particular, the stability margin agrees well with the URANS results. Moreover, the formulation has been able to reproduce the impact of geometric changes (in terms of inlet and outlet blade metal angles $\beta_{s q, 1}$ and $\beta_{s q, 2}$ and chord length $c$ ) when compared with mixing plane "meshed blades" computations. Improvements of the formulation could consist in improving the stagnation temperature ratio prediction. This requires to add modeling of what can increase the blade loading (in other words, what can locally induce additional deviation within the row). This can involve blade flow separations, shocks, tip gap effects, etc. Indeed, the formulation at stake only induces deviation following the blade metal angle $\beta_{s q}$, which is a good assumption in subsonic conditions far from the hub and shroud walls, but becomes more hazardous in transonic conditions or when subject to secondary flows.

The formulation has then been tested in an optimization process involving a design domain defined by six parameters: the inlet and outlet blade metal angles $\beta_{s q, 1}$ and $\beta_{s q, 2}$ and the chord length $c$ at two relative heights $(h=50 \%$ and $h=100 \%) .320$ points following a LHS have been computed in distorted conditions in a relative short amount of time (several days on 128 cores). This amount of evaluations was enough to build a metamodel on which a NSGA2 algorithm has enabled to identify optimum candidates (i.e. a Pareto front). However, the process has been slowed down by the necessity to assess the stability margin for each point as the body-force formulation at stake cannot predict the positive slope of the fan characteristic. It has been decided to interpolate these values from the domain extremities which have been assessed by "meshed blades" RANS computations. This process is quite a drawback, which highlights the necessity to improve the body-force formulation in order to intrinsically assess the stability limit. Indeed, through this process, several approximations and assumptions have been made and are summarized hereafter:

(i) The stability limits are extracted from RANS computations which are known to lack accuracy to detect stall inception compared to higher fidelity methods or experiments (ex. DES, LES) [40,41]. Besides, interpolations based on the domain extremities bring additional approximation and can bring discredit upon the stability margin prediction (as emphasized by the assessment of the selected optimized design).

(ii) The stability margin defined by equation (7) assumes that the stability limit does not depend on local inlet conditions and thus remains the same in the performance map $\left(p_{i} / p_{i, 0}\right)$ vs. $\dot{m}_{\text {corr }}$ i.e. $S$ remains the same. The stability margin is therefore only affected by variations of local working conditions due to the inlet distortion (i.e. at specific circumferential locations) i.e. the displacement of $M$ in the performance map (cf. FIGURE 10). Besides, this definition is very conservative as it implies that a blade only needs to reach stall conditions at a specific circumferential location for the entire row to be considered in stall conditions, which has been demonstrated not to be always the case [13].

Nevertheless, results have shown that geometric variation impacts are qualitatively consistent. An URANS simulation of an identified candidate showing an optimal combinaison of efficiency and stability margin has shown that strong discrepencies remain but that the design is indeed more robust to inlet distortion. Additional quantitative assessment are nonetheless required in order to completely assess the precision of the presented methodology. Moreover, several other assumptions have been made. In particular, the working conditions (massflow rate, stagnation pressure ratio) have not been conserved systematically between assessed points. In addition, multi-disciplinary constraints, such has mechanical robustness, have not been taken into account. Finally, this study has been focused solely on the fan blades. It could be interesting to extend this approach to nonaxisymetric OGV as their potential have been identified by many studies as good candidates to answer the issue of fan stage robustness to inlet distortion $[17,19,42]$. 


\section{ACKNOWLEDGMENT}

We would like to thank Safran Aircraft Engines for the funding, for the supporting and for sharing valuable data without which this research work would not have been possible. We are also greatly indebted to William Thollet and Guillaume Dufour who share with us their knowledge in body-force modeling methods. Finally, we would like to thank Itham Salah El Din for his technical support and advice on the optimization set-up.

\section{REFERENCES}

[1] Peters, A., 2014. "Ultra-short nacelles for low fan pressure ratio propulsors". PhD thesis, MIT, February.

[2] Thollet, W., 2017. "Body-force modeling of fanairframe interactions". $\mathrm{PhD}$ thesis, Institut Supérieur de l'Aéronautique et de l'Espace.

[3] Cao, T., Vadlamani, N. R., Tucker, P. G., Smith, A., Slaby, M., and Sheaf, C. T., 2016. "Fan-intake interactions under high incidence". Journal of Engineering for Gas Turbines and Power.

[4] Colin, Y., 2008. "Simulation numérique de la distorsion générée par une entrée d'air de moteur civil par vent de travers". PhD thesis, Ecole Nationnale Supérieure de l'Aéronautique et de l'Espace.

[5] Peters, A., Spakovszky, Z. S., Lord, W. K., and Rose, B., 2014. "Ultrashort nacelles for low fan pressure ratio propulsors". Journal of Turbomachinery, 137(TURBO-14-1120), July.

[6] Plas, A., Sargeant, M., Madani, V., Crichton, D., Greitzer, E., Hynes, T., and Hall, C., 2007. "Performance of a boundary layer ingesting (bli) propulsion system". In 45th American Institute of Aeronautics and Astronautics Aerospace Sciences Meeting and Exhibit, Reno, NV, January, pp. 811.

[7] Hardin, L., Tillman, T., Sharma, O. P., Berton, J., and Arend, D. J., 2012. "Aircraft system study of boundary layer ingesting propulsion". AIAA, 3993, p. 2012.

[8] Liou, M.-S., and Lee, B. J., 2012. "Minimizing inlet distortion for hybrid wing body aircraft". Journal of Turbomachinery, 134(3), p. 031020.

[9] Florea, R. V., Matalanis, C., Hardin, L. W., Stucky, M., and Shabbir, A., 2015. "Parametric analysis and design for embedded engine inlets". Journal of Propulsion and Power.

[10] Liou, M.-F., Kim, H., Lee, B., and Liou, M.-S., 2017. "Aerodynamic design of integrated propulsion-airframe configuration of the hybrid wing-body aircraft". In 35th AIAA Applied Aerodynamics Conference.

[11] Cousins, W. T., Georges, M. J., and Rezaei, H., 2003. "Inlet distortion testing and analysis of a high-bypass ratio turbofan engine". ISABE Paper(2003-1110).

[12] Ferrar, A. M., 2015. "Measurement and uncertainty anal- ysis of transonic fan response to total pressure inlet distortion". $\mathrm{PhD}$ thesis.

[13] Perovic, D., Hall, C. A., and Gunn, E. J., 2015. "Stall inception in a boundary layer ingesting fan". In Proceedings of ASME Turbo Expo 2015: Turbine Technical Conference and Exposition.

[14] Fidalgo, V. J., Hall, C., and Colin, Y., 2012. "A study of fan-distortion interaction within the nasa rotor 67 transonic stage". Journal of Turbomachinery, 134(5), p. 051011.

[15] Sheoran, Y., Bouldin, B., Hoover, R., and Matwey, M., 2017. "A centrifugal compressor operability correlation with combined total pressure and swirl distortion". In ASME Turbo Expo 2017: Turbomachinery Technical Conference and Exposition, American Society of Mechanical Engineers, pp. V001T01A015-V001T01A015.

[16] Frohnapfel, D. J., Lowe, K. T., and O'Brien, W. F., 2018. "Experimental quantification of fan rotor effects on inlet swirl using swirl distortion descriptors". Journal of Engineering for Gas Turbines and Power, 140(8), p. 082603.

[17] Hall, D. K., 2015. "Analysis of civil aircraft propulsors with boundary layer ingestion.”. PhD thesis, MIT.

[18] Gunn, E., and Hall, C., 2014. "Aerodynamics of boundary layer ingesting fans". In ASME Turbo Expo 2014: Turbine Technical Conference and Exposition, American Society of Mechanical Engineers, pp. V01AT01A024V01AT01A024.

[19] Gunn, E., and Hall, C., 2017. "Non-axisymmetric stator design for boundary layer ingesting fans". In ASME Turbo Expo 2017: Turbomachinery Technical Conference and Exposition, American Society of Mechanical Engineers.

[20] Arend, D. J., 2018. "Experimental evaluation of bli propulsion: The boundary layer ingesting inlet/distortion-tolerant fan solution". In AIAA Propulsion and Energy Forum, Cincinnati, $\mathrm{OH}$.

[21] Hardin, L. W., Cousins, W. T., Wolter, J. D., Arend, D. J., and Hirt, S. M., 2018. "Data analysis techniques for fan performance in highly-distorted flows from boundary layer ingesting inlets". In 2018 AIAA Aerospace Sciences Meeting, p. 1888.

[22] Provenza, A. J., Duffy, K. P., and Bakhle, M. A., 2019. "Aeromechanical response of a distortion-tolerant boundary layer ingesting fan". Journal of Engineering for Gas Turbines and Power, 141(1), p. 011011.

[23] Thollet, W., Dufour, G., and Carbonneau, X., 2016. "Assessment of body force methodologies for the analysis of intake-fan aerodynamic interactions". In Proceedings of ASME Turbo Expo 2016.

[24] Godard, B., De Jaeghere, E., Nasr, N. B., Marty, J., Barrier, R., and Gourdain, N., 2017. "Methodologies for turbofan inlet aerodynamics prediction”. In 35th AIAA Applied Aerodynamics Conference, p. 3413.

[25] Gong, Y., 1999. "A computational model for rotating stall 
and inlet distortions in multistage compressors". PhD thesis, Massachusetts Institute of Technology, Dept. of Aeronautics and Astronautics.

[26] Guo, J., Hu, J., and Yin, C., 2015. "A three-dimensional analysis code of compressor performance and stability for steady inlet distortion”. In Proceedings of ASME nTurbo Expo 2015: Turbine Technical Conference and Exposition.

[27] Thollet, W., Blanc, F., Dufour, G., and Carbonneau, X., 2015. "Body force modeling for aerodynamic analysis of intake-fan interactions". In 50th 3AF Internationnal Conference on Applied Aerodynamics.

[28] Godard, B., 2018. "Etude et méthodologies de simulation de doublet entrée d'air - soufflante pour la conception de turbofan de nouvelle génération". $\mathrm{PhD}$ thesis, Université de Toulouse - Institut Supérieur de l'aérodynamique et de l'Espace.

[29] Kottapalli, A. P., 2013. "Development of a body force model for centrifugal compressors". PhD thesis, Massachusetts Institute of Technology.

[30] Hall, C., and Greitzer, E.and Tan, C., 2016. "Analysis of fan stage design attributes for boundary layer ingestion.”. In ASME Turbo Expo.

[31] Marble, F. E., 1964. "Three-dimensional flow in turbomachines". High Speed Aerodynamics and Jet Propulsion, 10, pp. 83-166.

[32] Cambier, L., Heib, S., and Plot, S., 2013. "The onera elsa cfd software: input from research and feedback from industry". Mechanics \& Industry, 14(3), pp. 159-174.

[33] Roe, P. L., 1981. "Approximate riemann solvers, parameter vectors, and difference schemes". Journal of computational physics, 43(2), pp. 357-372.

[34] Smith, B., 1990. “The k-kl turbulence model and wall layer model for compressible flows". In AIAA, Fluid Dynamics, Plasma Dynamics and Lasers Conference, 21 st, Seattle, WA, p. 1990.

[35] NUMECA, 2008. "User manualautogrid5 release 8.4 : A full automatic hexahedral grid generator for all types of rotating macmanual".

[36] Benoit, C., Péron, S., and Landier, S., 2015. "Cassiopee: a cfd pre-and post-processing tool". Aerospace Science and Technology, 45, pp. 272-283.

[37] Dunham, J., 1965. "Non-axisymmetric flows in axial compressors". Mechanical Engineering Science Monograph, MESM.

[38] McKay, M. D., Beckman, R. J., and Conover, W. J., 1979. "Comparison of three methods for selecting values of input variables in the analysis of output from a computer code". Technometrics, 21(2), pp. 239-245.

[39] Deb, K., Agrawal, S., Pratap, A., and Meyarivan, T., 2000. "A fast elitist non-dominated sorting genetic algorithm for multi-objective optimization: Nsga-ii”. In Parallel Problem Solving from Nature PPSN VI, M. Schoenauer, K. Deb,
G. Rudolph, X. Yao, E. Lutton, J. J. Merelo, and H.-P. Schwefel, eds., Springer Berlin Heidelberg, pp. 849-858.

[40] Gourdain, N., $2005 . \quad$ "Simulation numérique des phénomnes de décollement tournant dans les compresseurs axiaux". PhD thesis, Ecole Centrale de Lyon.

[41] Choi, M., Vahdati, M., and Imregun, M., 2011. "Effects of fan speed on rotating stall inception and recovery". Journal of Turbomachinery, 133(4), p. 041013.

[42] Kumar, S., Turner, M. G., Siddappaji, K., and Celestina, M., 2018. "Aerodynamic design system for nonaxisymmetric boundary layer ingestion fans". In ASME Turbo Expo 2018: Turbomachinery Technical Conference and Exposition, American Society of Mechanical Engineers. 